WIENER SLAVISTISCHES JAHRBUCH, Band 57/2011, 131-138

(C) 2011 by Österreichische Akademie der Wissenschaften, Wien

Milan Minal JeVí́

\title{
Der Dual im Kroatisch-Kirchenslavischen
}

Bekanntlich ist in den Systemen, die drei Numeri - Singular, Plural und Dual unterscheiden, der Dual doppelmarkiert. Gegenüber dem Singular ist er ein NichtSingular, der „mehr als eins“ gegenüber „eins“ bedeutet, und gegenüber dem Plural ist er ein Nicht-Plural, der „Zwei“ gegenüber „mehr als zwei“ bedeutet. ${ }^{1}$ Daraus ergeben sich folgende Implikationen: 1. Eine Sprache ohne Singular kann keinen Plural haben und 2. eine Sprache ohne Plural kann keinen Dual haben. Deswegen verwundert es nicht, dass der Dual weltweit viel seltener vorkommt als Singular und Plural. Nach G. Corbetts (2000: 281) statistischen Angaben zum Slowenischen, Obersorbischen und Sanskrit stehen zwischen 65 und 75\% der Beispiele im Singular, zwischen 25 und 30\% im Plural und nur zwischen 0,5 und 6\% im Dual. Ähnlich verhält es sich im Kroatisch-Kirchenslavischen. Dass der Dual doppelmarkiert ist, bringt es mit sich, dass er im Gegensatz zu den semantisch offenen, unbegrenzten und inklusiven anderen beiden Numeri eine geschlossene, maximal begrenzte und exklusive grammatische Kategorie darstellt. Er betont die Zusammengehörigkeit zweier Objekte und auch ihre Zweiheit, letzteres allerdings angesichts des sprachlichen oder außersprachlichen Kontexts großteils redundant. Dies alles macht in solchen Systemen den Dual zur instabilsten numeralen Kategorie, weshalb er in meisten Sprachen auch die Tendenz zum Verschwinden zeigt.

Über den Untergang des slavischen Duals gibt es eine reichhaltige Literatur. Wir wissen heute, dass er ein Jahrhunderte währender komplizierter Prozess war und dass verschiedene Dualformen verschiedenes Schicksal hatten. Der urslavische Dual hatte zwei Grundbedeutungen: Paarigkeit und Zweiheit. Er konnte sowohl paarige Objekte, vor allem Körperteile, als auch gewöhnlich nicht in Paaren auftretende beliebige zwei Objekte bezeichnen. Aleksandar Belić (1932) unterscheidet im Slavischen den freien vom gebundenen Dual. Beim freien Dual könne derjenige, der sich auf ein Paar bezieht, vom distributiven, der sich auf mehrere Paare bezieht, unter-

${ }^{1}$ Vgl. Naylor 1972 und Lenček 1982. 
schieden werden. Der gebundene Dual ist vom Numerale $d v a$ (oder $o b a$ ) abhängig, ob es nun explizit ausgedrückt ist oder nicht. Er kann sich auf beliebige zwei Gegenstände beziehen. Das Schicksal des freien und des gebundenen Duals in der Entwicklung der slavischen Sprachen einschließlich des Kroatisch-Kirchenslavischen ist verschieden. Typologisch und vergleichend-historisch sind noch weitere Dualkategorien zu unterscheiden. ${ }^{2}$ Wir folgen hier Žolobov und Krys'ko (2001), die neben dem freien und dem gebundenen auch den pronominal-verbalen Dual, den Dual in Konstruktionen mit zwei Substantiven (bzw. Namen) und den kongruenten Dual verzeichnen. Frühere Forscher ${ }^{3}$ haben geglaubt, dass der freie Dual schon in vorhistorischer Zeit (also im Urslavischen) verloren gegangen ist. Heute wissen wir, dass der freie Dual früher als der gebundene, aber nicht vor dem 13. Jahrhundert geschwunden ist. André Vaillant hatte schon vermutet, und Žolobov (1997) und Večerka (2000) haben es klar gezeigt, dass man das Abwechseln von Dual- und Pluralformen nicht als Zeichen des Verlustes des Duals als Kategorie werten darf.

Die Prager Schule unterscheidet drei Phasen im Untergang einer grammatischen Kategorie. Die erste ist der Relevanzverlust, nach welchem in den markierten Positionen das unmarkierte Glied der Opposition das markierte ersetzen kann, nicht aber umgekehrt. In unserem Fall bedeutet dies, dass die Pluralformen im Dualkontext vorkommen können, nicht jedoch die Dualformen im Pluralkontext. Obwohl die markierte und unmarkierte Form abwechseln, ist die Opposition zwischen ihnen nicht aufgehoben. Die Formen variieren nicht frei, sondern sind sie partial komplementär verteilt. Die zweite Phase ist der Funktionsverlust, nach welchem die Opposition aufgehoben ist und die Formen frei variieren. In der dritten Phase (zu der es in unserem Fall nicht gekommen ist) geht die markierte Form verloren. Diese Phase nennen wir Formverlust.

Was das Kroatisch-Kirchenslavische betrifft, so wechseln schon in den ältesten Denkmälern der so genannten Übergangsperiode (12. und 13. Jahrhundert) distributiver Dual und Plural miteinander ab, wie folgende Beispiele belegen:

(1) a. i obuvše nozê vb ugotovanie eva(n)ĵelie miru - Fg Mih 2c (E 6, 15)

b. prinese cênu : ï položi prêdъ nogama ap(usto)lomı - Fg Grš 1r

c. i prinesъ čъstъ eteru : pri nogahъ ap(usto)lomъ položi - Fg Grš 1r

d. î vъzložiše ruki svoe na ap(usto)ly - Fg Grš $1 \mathrm{v}$

${ }^{2}$ Holzer 2010 unterscheidet in den indogermanischen Sprachen insgesamt vier Typen des Dualgebrauchs: 1. der individuale homogene Typ, in welchem der Dual zwei gleichartige Objekte bezeichnet, 2. der individuale heterogene Typ, in welchem der Dual zwei verschiedenartige Objekte bezeichnet, 3. der kollektivische homogene Typ, in welchem der Dual zwei gleichartige Kollektive bezeichnet und 4. der kollektivische heterogene Typ, in welchem der Dual zwei verschiedenartige Kollektive bezeichnet. Er meint aber, dass diese Bedeutungsvielfalt nur scheinbar ist und im Urindogermanischen hinter ihr nur ein einziges gedankliches Konzept stand.

3 Belić 1932, Iordanskij 1960 und besonders Dostál 1954. 
Solche Fälle kommen auch in den kanonischen Texten vor. Da es aber keine Beispiele der Verwendung des Duals im Pluralkontext gibt, kann nicht behauptet werden, dass der freie Dual verloren gegangen wäre.

Im 14. und 15. Jahrhundert steht in den liturgischen Büchern der freie Dual nur dann, wenn es sich um die Hände oder Füße eines oder zweier Menschen handelt. Sind es mehr als zwei Menschen, steht gewöhnlich der Plural. Das lässt sich anhand folgender Beispiele illustrieren:

(2) a. ne umivaût' bo ruk' svoih' egda êdet' - M Vat 4 48a

b. da noge sebê meû soboû umiûtb - M Nov $89 \mathrm{~d}$

c. izbavitı i ot rukb našihb $-\mathrm{Br} \mathrm{VO} 224 \mathrm{a}$

d. i vsa položi k nogamb ap(usto)lb - Br Lab 165b

e. g(ospod)i ne takmo lê nozê moi na i rucê i gl(a)vu ... aĉe azb g(ospod)b i moistarb umihь nogi v(a)mь koliko veĉe dlžni este drugь drugu umivati nozê $-\mathrm{Br}$ VO 237a

f. d(u)hb životın' ot b(og)a vnidet' v na i staneta na nogu svoeû - Br VO 272c

g. vzdvigb rucê svoi $\mathrm{k} n(\mathrm{e})$ besi i p(o)m(o)li se $-\mathrm{Br} \mathrm{Vat}_{6} 209 \mathrm{a}$

h. krila imuĉa sv(ê)tla i zrakb plamenan' svateĉ' se · d'vê krunê v' ruku noseĉb Br Lab 157c

Sehr selten trifft man auf Dualformen bei mehr als zwei Paaren:

(3) a. luče estı mnê vpasti v rucê č(lovê) $\mathrm{kb}-\mathrm{Br} \mathrm{VO} 432 \mathrm{~d}$

b. s nêčistivimi ... v nihže ruku bêzakoniê sutb - Ps Lob $15 \mathrm{v}$

c. se êko oči rab' v ruku g(ospod)b svoih' - M Vat 4 44d

d. se predaet' se s(i)nь č(lovêč)ski v rucê grêšnih' - M Nov $80 \mathrm{~d}$

und ganz ausnahmsweise auf Pluralformen bei einem Paar:

(4) tebê zališna sutb · na g(ospod)nimb nogamb potrêbna sutb - Br VO 226a

Es gibt auch Beispiele, in denen beiderlei Formen gemeinsam erscheinen:

(5) a. večši manšimi umiûts nozi i otaru i celuûtb nogi ihь - Br VO 236a

b. rucê že v'sagda nose pokr'veni i nogami ot tolê hoêše obuvenimi - $\mathrm{Br} \mathrm{Vat}_{6}$ $190 \mathrm{a}$

Das ist die Situation in den liturgischen Büchern bis zur zweiten Hälfte des 15. Jahrhunderts. In den Brevieren vom Ende des 15. Jahrhunderts ${ }^{4}$ sind Plural und Dual auch für ein oder zwei Paare frei austauschbar und können mit dem Verb und dem Pronomen im Plural oder im Dual kongruieren:

\footnotetext{
${ }^{4}$ Zum Beispiel im Zweiten Brevier von Novi Vinodolski aus dem Jahr 1495.
} 
(6) $a{ }^{5} d(u) h ь$ života ot' b(og)a poslanь vnide v niü i stasta na nogahь svoihь $-\mathrm{Br} \mathrm{N}_{2}$ $137 \mathrm{a}$

b. i vžeda i(su)sb s(ve)ti rukami čav'li prigvoždenь i nogami - $\mathrm{Br} \mathrm{N}_{2} 130 \mathrm{c}$

c. i kada priês'ta hlêba polb vs(a)komu v rukahb osta $-\mathrm{Br} \mathrm{N}_{2} 364 \mathrm{c}$

Pluralformen kamen in dieser Rolle jetzt viel häufiger als im 14. Jahrhundert vor.

In den nichtliturgischen Sammelbänden (zbornici) verhält es sich etwas anders. Im ältesten, dem stark kroatisierten Pariser Sammelband aus dem Jahr 1375, verwendet der Schreiber systematisch auch dann den Plural, wenn es sich um ein Paar von Objekten handelt:

(7) a. i dêla ruk' tvoih' suts $n(e) b(e) s a-C$ Par $88 r$

b. o(t)če v ruke tvoe priporučuû d(u)hь moi - C Par 204

c. ta istrgnet' ot' sêti noge moe $-C$ Par $24 \mathrm{v}$

d. po čavli v'bienie rukamb i nogamb ego - C Par 207r

Nur vereinzelt kommen hier noch die Dualformen vor, und zwar vor allem in den Evangelien. Wahrscheinlich war also der freie Dual zumindest in einigen südlichen kroatischen Dialekten schon verloren gegangen. In den Sammelbänden aus dem 15. Jahrhundert variiert die Situation von Band zu Band und von Text zu Text. In den stärker kroatisierten Texten kommt der Dual seltener vor, in den besser tradierten tritt er auch in distributiver Bedeutung auf. ${ }^{6}$ Generell kann man sagen, dass in diesen Texten Plural und Dual unabhängig davon, ob es sich um ein Paar oder um mehrere Paare von Objekten handelt, frei variieren. Das lässt sich anhand von Beispielen aus dem Ivančić-, dem Oxforder, dem Petris-, dem Berčić-Sammelband und dem Sammelband der Kroatischen Akademie der Wissenschaften und Künste illustrieren:

(8) a. s' nogami se h(rbsto)vimi v'mêsti - C Ivan 113a

b. po čav'li v'bodenie rukama i nogama ego - C Ivan 140r

c. do večera že v'sadiše ga v' tam'nicu s'vezavše emu r(u)ke i noge - C Bč 40r

d. rucê i nozê uvênuše i v' grobs polagaût' me - C Bč 33b

e. ê bo kada umih' noge nega razumihь k(a)ko ti es'ta nozi ki on'da umih' pod' dubom' mam'breiskim' - C Oxf 14c

f. i bêhu tu č(lovê)ci živucii imêhu lica kako vol' rozi elêni nozi koz'e črêsla ovna - C Pet $229 \mathrm{v}$

g. tada tatom ruki lûto vstrepeĉu $-\mathrm{C}$ Pet $345 \mathrm{r}$

h. obêsiti ga za noge - C Ac 94r

i. prokleti ruci vaši kimi mnoga ubiistva i krviprolitiê ... stvorili este - C Ac 71r

${ }^{5}$ Das ist ein Parallelbeispiel zu (2f).

${ }^{6}$ Zum Beispiel steht im Petris-Sammelband gewöhnlich der Dual für ein Paar von Objekten, aber wenn es sich um mehr als nur ein Paar handelt, wechseln sich Dual und Plural frei ab. 
Aus typologischen Gründen liegt die Annahme nahe, dass der Untergang des Duals an der Spitze der Belebtheitshierarchie beginnt, also bei den Pronomina der ersten und der zweiten Person. Den Grammatiken ist zu entnehmen, dass schon in kanonischen altkirchenslavischen Texten Plurale anstelle von Dualen auftauchen. Auf die kroatische Redaktion trifft dies von deren Beginn an zu. Zum Beispiel erscheint in den Krker Passionalfragmenten von der Mitte des 13. Jahrhunderts einige Male die Akkusativform $n i$ anstelle von $n a$ im Dualkontext und einmal im Instrumental die Pluralform s nami anstelle der Dualform s nama:

(9) a. ona že obeĉasta se ubiti ni i vzeti prêl'stnoe zlato - Fg Period Bb

b. ona že zaušista iv(a)na i vlêsta ni ne v' tbmnicu nь v dom' ukrom' stoeĉa êko pobiti ni hoteĉa - Fg Period Bc

c. î si g(lago)lûcu ivanu kb mnê pridosta voina i esta ni dioskorid' že ne bê s nami - Fg Period Bc

d. ace obrativê se $\mathrm{k}$ dioskoridu inako g(lago)lûcea to ispitavı zlê na ni vstanet' i izgubit ni - Fg Period Bd

Doch vertritt in den liturgischen Texten bis zum Ende der kirchenslavischen Periode der Plural des Personalpronomens nur sehr selten den Dual. Unter den Stichwörtern $m i$ und $v i$ in der Kartothek des Rječnik crkvenoslavenskoga jezika hrvatske redakcije im Staroslavenski institut in Zagreb sind nicht mehr als 15 solche Fälle verzeichnet. In allen Beispielen, in denen das Pronomen Subjekt ist, steht das Verb im Dual. Zum Beispiel:

(10) a. ${ }^{7}$ mi ubo v' prav'du dostoina po dêlomb naû vspriemlevê - M Nov $87 \mathrm{a}$

b. ${ }^{8}$ mi krst'ênina esvê stvorena - $\mathrm{Br} \mathrm{Vat}_{6} 133 \mathrm{c}$

c. mi usnusva snom veliem i pridosta $\tilde{b}$ orla $-\mathrm{C}$ Tk $91 \mathrm{v}$

Hier sind einige Beispiele mit Genitiv Dual anstelle des Akkusativs besonders interessant:

(11) a. nь molû vaû druga dobraê p(o)m(i)luita stranstvo moe - Fg Pass $2 b$

b. ace kto uprosit' vaû rbcêta dêlo g(ospod)ne est' $-\mathrm{M} \mathrm{Vat}_{4} 71 \mathrm{~d}$

c. idita v' gradı i usrêceet' vaû č(lovê)kb baril' vode nose - C Par 247v

Die Ausbreitung der Belebtheitskategorie auf den Dual belegt, dass der Dual noch eine lebendige Kategorie war. Es sei darauf hingewiesen, dass es in den kroatischkirchenslavischen Texten kein Beispiel der zweiten Person Dual va, die gewöhnlich in den altkirchenslavischen Grammatiken angeführt ist, gibt, sondern nur die mit

\footnotetext{
${ }^{7} \mathrm{M} \mathrm{Vat}_{4} 86 \mathrm{~b}$ hat hier den Dual: vê ubo v pravdu dostoina po dêlom' nâ̂ vspriemlevê.

${ }^{8} \mathrm{Br} \mathrm{N}_{2} 413 \mathrm{~d}$ hat hier den Dual: vê h(rbst) bêna esvê stvorena.
} 
dem Plural identische Form vi. Das bedeutet, dass hier das Kroatisch-Kirchenslavische den urslavischen Zustand bewahrt hat. ${ }^{9}$

Im Korpus habe ich nur zwei Beispiele gefunden, in denen die Nominalphrase mit dem Numerale $d v a$ im Plural statt im Dual steht. Das erste stammt aus dem $B a$ romić-Brevier aus dem Jahr 1493, das zweite aus dem Žgombić-Sammelband aus dem 16. Jahrhundert:

(12) a. kada se zn(a)m(e)nanь d'vimi meči êvi - Br Bar 422b

b. i se pridoše $\tilde{\ddot{1}} \cdot$ anĵ(e)la i dve sile nebeske $-\mathrm{C} \breve{Z} g$ 34v

Interessant ist, dass in beiden Fällen auch $d v a$ im Plural auftritt. Mit dem Numerale $o b a$ gibt es kein solches Beispiel. Wahrscheinlich war damals in einigen kroatischen Dialekten der Dual als Kategorie schon verloren gegangen. Das gilt selbstverständlich nicht für das Kroatisch-Kirchenslavische.

In den kroatisch-kirchenslavischen Denkmälern habe ich kein Beispiel des im Altrussischen belegten doppelten Duals, also von zwei Namen, die wie im Götterdvandva beide im Dual stehen, und auch keines für den so genannten elliptischen Dual, bei dem ein Name im Dual das ganze Paar vertritt, gefunden. Für die von Belić sindetska dvojina genannte Konstruktion aus zwei mit der Konjunktion $i$ verbundenen Nominalphrasen im Singular gibt es in unserem Korpus nur einige Beispiele. Hier ist aber zu erwähnen, dass in solchen Konstruktionen, wenn koordinierte Nominalphrasen Subjekt sind, in vielen Sprachen das Verb entweder mit beiden Nominalphrasen oder aber nur mit einer kongruiert, ${ }^{10}$ also nur im Singular oder im Dual stehen kann. Tatsächlich gibt es einige Beispiele mit dem Verb im Dual:

(13) a. az že i otročiĉ́ si doidevê do sadê i poklonša se v'zv'rativêse k vamb - M Nov 101d (dasselbe auch im Br VO 145d)

b. adamb $\mathrm{v}$ istinu i žena ego $\cdot$ ne stidêšeta $\mathrm{se}-\mathrm{Br} \mathrm{Vb}_{2} 3 \mathrm{~d}$

Aus den bisher angeführten Beispielen ist ersichtlich, dass im Dualkontext ein Verb im Dual wie im Plural zusammen mit einem Subjekt im Dual wie im Plural auftreten kann. Auch Pronomina, die sich auf Nominalphrasen im Dual oder auf zwei mit der Konjunktion $i$ verbundenen Nominalphrasen beziehen, können im Dual oder im Plural stehen:

(14) a. i udari têlo v nozê želêznê i skudêlnê i stni e do konca - Br VO 439a

b. muža i ženu stvori ê - $\mathrm{Br} \mathrm{Vb}_{2} 2 \mathrm{c}$

${ }^{9}$ Nach Decaux 1953, Vaillant 1958: 456-459 und Žolobov - Krys'sko 2001: 75-78 lautete die zweite Person Dual urslavisch nur vy. Diese Form ist vom kroatischen (und kroatischkirchenslavischen) $v i$ fortgesetzt. Die Form $v a$ sei in Analogie zum Akkusativ entstanden.

10 Vgl. Mihaljević 2002: 333. 
So zeigt es sich, dass der Dual im Kroatisch-Kirchenslavischen die ganze Periode hindurch eine lebendige grammatische Kategorie war. Anfänglich hatte er alle Bedeutungen, die er im Altkirchenslavischen hatte. Später erlitt er zwar den Relevanzverlust, nicht jedoch den Funktionsverlust. Obwohl sich Dual- und Pluralformen, wenn auch nur sporadisch, im Dualkontext abwechseln, gibt es keinen Fall einer Verwendung des Duals im Pluralkontext. Zum Beispiel erscheinen im Genitiv Plural der Substantive ruka und noga nur die alten Pluralformen rukb und nogb und nie die Dualformen ruku und nogu wie im heutigen Kroatischen. Auch auf die Numeralia tri und četire hat sich der Dual nicht ausgebreitet; mit ihnen steht das Substantiv immer im Plural.

$$
\begin{gathered}
\text { Abkürzungsverzeichnis zu den } \\
\text { kroatisch-glagolitischen Handschriften }
\end{gathered}
$$

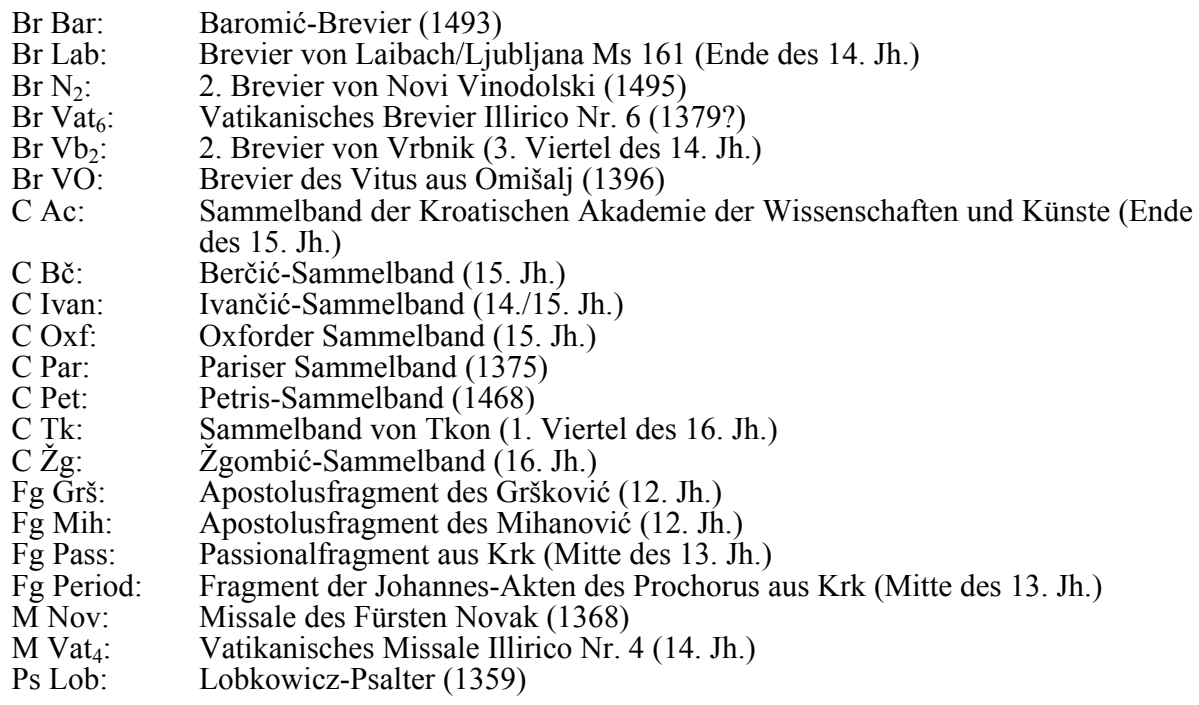

\section{Literatur}

Belić 1932:

Corbett 2000:

Decaux 1953:

Derganc 1993:

Derganc 1998:

Dostál 1954:

Holzer 2010:
Aleksandar Belić, O dvojini u slovenskim jezicima, Beograd Greville G. Corbett, Number, Cambridge

Étienne Decaux, Le nominatif duel des pronoms personnels polonais, Revue des études slaves 30, 95-97

Aleksandra Derganc, Spremembe nekaterih dvojinskih oblik in zvez v slovenščini in ruščini, Slavistična revija 41, 209-218

Aleksandra Derganc, Nekatere paralele v razvoju dvojine v slovenščini in lužiški srbščini, Slavistična revija 46, 45-54

Antonín Dostál, Vývoj duálu v slovanských jazycích, zvláště v polštině, Praha

Georg Holzer, Zum Gebrauch der Numeri im Indogermanischen, Finnisch-Ugrische Mitteilungen 32/33 (2008/2009 = Gedenkschrift für Eugen A. Helimski. Herausgegeben von Valentin Ju. Gusev und Anna 
Widmer unter redaktioneller Mitarbeit von Annkatrin Klein), Hamburg 2010, 189-206

Iordanskij 1960: $\quad$ A. M. Iordanskij, Istorija dvojstvennogo čisla v russkom jazyke, Vladimir

Kuzmić 2006: $\quad$ Boris Kuzmić, Odnos između dvojine i množine u predstandardnom razdoblju hrvatskoga jezika, Doktorska disertacija, Filozofski fakultet Sveučilišta u Zagrebu, Zagreb

Lenček 1982:

Rado Lenček, On Poetic Functions of the Grammatical Category of Dual, South Slavic and Balkan Linguistics (= Studies in Slavic and General Linguistics 2), Amsterdam, 193-214

Lötzsch 1965: R. Lötzsch, Die spezifischen Neuerungen der sorbischen Dualflexion, Budyšin

Mareš 1977:

František Václav Mareš, Duál slovinského a lužickosrbského slovesa,

Mihaljević 2002:

in: Franc Jakopin (Red.), Nahtigalov zbornik, Ljubljana 201-214

Milan Mihaljević, The Structure of Croatian Alternative Questions, in: Peter Kosta, Jens Frasek (Hrsg.), Current Approaches to Formal Slavic Linguistics, Frankfurt am Main

Naylor 1972:

Kenneth E. Naylor, On Some Developments of the Dual in Slavic, International Journal of Slavic Linguistics and Poetics 15, 1-8

Vaillant 1958: $\quad$ André Vaillant, Grammaire comparée des langues slaves. T. II. Morphologie. 2. pt.: Flexion pronominale, Lyon - Paris

Žolobov 1998: $\quad$ Oleg F. Žolobov, Symbolik und historische Dynamik des slavischen Duals, Frankfurt am Main

Žolobov - Krys'ko 2001: Oleg F. Žolobov, Vadim B. Krys'ko, Istoričeskaja grammatika drevnerusskogo jazyka: Dvojstvennoe čislo, Moskva

A b s tra c t: Dual in Croatian Church Slavonic. The author describes the use and development of the dual in Croatian Church Slavonic. He shows that in Croato-Glagolitic liturgical texts the dual was an operative category during the whole Church-Slavonic period (until the middle of the $16^{\text {th }}$ century). It was used regularly whenever two entities were involved. In the so-called transitional period (the $12^{\text {th }}$ and $13^{\text {th }}$ centuries), the dual had both of its Common Slavic meanings, i.e. the meaning of a pair as well as the meaning of two, although in the texts from that period the distributive dual alternates already with the plural. Later, the dual gradually lost the meaning of a pair and preserved only the meaning of two. In the texts from the $14^{\text {th }}$ and $15^{\text {th }}$ centuries the scribes used free dual only for one or two pairs of entities. For instance, if they spoke about the hands of one or two men, they used the dual, and if they spoke about the hands of three or more men, they used the plural. By the end of the $15^{\text {th }}$ century, the loss of free dual had been completed. After that, the dual alternated freely with the plural even in cases when it referred to one or two pairs of entities. The dual was consistently used only with the numbers $d v a$ 'two' and $o b a$ 'both'. In the corpus, there are only two examples in which the dual has been replaced by the plural when the number two has been explicitly mentioned. The author concludes that in Croatian Church Slavonic we can only speak about the loss of relevance, and not about the loss of the function of the dual. Although the dual and the plural alternate in dual contexts, there are no examples in which the dual occurs instead of the plural in the plural context. This is also true for the genitive plural of the nouns ruka 'hand' and noga 'foot' as well as for the numbers tri 'three' and četire 'four'. In the first case, only old plural forms rukb and nogb occur, and never the dual forms ruku and nogu, which are characteristic for modern Croatian. After the numbers tri and četire the nouns occur only in the plural and never in the dual form (as in modern Croatian).

Ke y w ords: Church Slavonic language, Croato-Glagolitic texts, grammatical categories, dual

Milan Mihaljević

Staroslavenski institut

Demetrova 11, 10000 Zagreb, Kroatien

mihalj@stin.hr 